\title{
ChemComm
}



Cite this: Chem. Commun., 2016, 52, 12905

Received 1st July 2016, Accepted 4th October 2016

DOI: $10.1039 / c 6 c c 05473 k$

www.rsc.org/chemcomm

\section{Multifaceted magnetization dynamics in the mononuclear complex $\left[\mathrm{Re}^{\mathrm{IV}} \mathrm{Cl}_{4}(\mathrm{CN})_{2}\right]^{2-} \dagger$}

\author{
Xiaowen Feng, ${ }^{a}$ Jun-Liang Liu, ${ }^{\text {bcd }}$ Kasper S. Pedersen, ${ }^{\text {cde }}$ Joscha Nehrkorn, $\ddagger^{f}$ \\ Alexander Schnegg, ${ }^{f}$ Karsten Holldack, ${ }^{g}$ Jesper Bendix, ${ }^{\text {h }}$ Marc Sigrist, ${ }^{i j}$ \\ Hannu Mutka, ${ }^{i}$ Dumitru Samohvalov, ${ }^{c d}$ David Aguilà, ${ }^{c d}$ Ming-Liang Tong, ${ }^{b}$ \\ Jeffrey R. Long*akl and Rodolphe Clérac*cd
}

The mononuclear complex $\left(\mathrm{Bu}_{4} \mathrm{~N}\right)_{2}\left[\mathrm{Re}^{\mathrm{IV}} \mathrm{Cl}_{4}(\mathrm{CN})_{2}\right] \cdot 2 \mathrm{DMA}$ (DMA = $\mathrm{N}, \mathrm{N}$-dimethylacetamide) displays intricate magnetization dynamics, implying Orbach, direct, and Raman-type relaxation processes. The Orbach relaxation process is characterized by an energy barrier of $39 \mathrm{~K}\left(27 \mathrm{~cm}^{-1}\right)$ that is discussed based on high-field electron paramagnetic resonance (EPR), inelastic neutron scattering and frequencydomain THz EPR investigations.

For paramagnetic molecules with a spin ground state of $S_{\mathrm{T}}$, the presence of anisotropy may result in a magnetic bistability through the stabilization of the $M_{S}=+S_{\mathrm{T}}$ and $-S_{\mathrm{T}}$ microstates, which are separated by an energy barrier, $\Delta$, thereby leading to slow dynamics of the magnetization. As a family, such molecules are referred to as single-molecule magnets (SMMs). ${ }^{1,2}$ Although the first generations of SMMs were polynuclear complexes characterized by ever larger

${ }^{a}$ Department of Chemistry, University of California Berkeley, California, 94720, USA. E-mail: jrlong@berkeley.edu

${ }^{b}$ MOE Key Lab of Bioinorganic and Synthetic Chemistry, School of Chemistry, Sun Yat-Sen University, Guangzhou 510275, P. R. China

${ }^{c}$ CNRS, CRPP, UPR 8641, F-33600 Pessac, France.

E-mail: clerac@crpp-bordeaux.cnrs.fr

${ }^{d}$ Univ. Bordeaux, CRPP, UPR 8641, F-33600 Pessac, France

${ }^{e}$ CNRS, ICMCB, UPR 9048, F-33600 Pessac, France

${ }^{f}$ Berlin Joint EPR Laboratory, Institut für Nanospektroskopie,

Helmholtz-Zentrum für Materialien und Energie, 12489 Berlin, Germany

${ }^{g}$ Helmholtz-Zentrum für Materialien und Energie, Inst. f. Methoden und

Instrumente der Forschung mit Synchrotronstrahlung, 12489 Berlin, Germany

${ }^{h}$ Department of Chemistry, University of Copenhagen, DK-2100 Copenhagen,

Denmark

Institut Laue-Langevin, 38042 Grenoble Cedex 9, France

${ }^{j}$ Institute of Chemistry, Academia Sinica, Nankang, 11529 Taipei, Taiwan, ROC

${ }^{k}$ Materials Sciences Division, Lawrence Berkeley National Laboratory, Berkeley,

California 94720, USA

${ }^{l}$ Department of Chemical and Biomolecular Engineering,

University of California Berkeley, Berkeley, California 94720, USA

$\dagger$ Electronic supplementary information (ESI) available: Experimental details, crystallographic data, and magnetic and spectroscopic characterization. CCDC 1488917. For ESI and crystallographic data in CIF or other electronic format see DOI: $10.1039 / \mathrm{c} 6 \mathrm{cc} 05473 \mathrm{k}$

\# Current address: Department of Chemistry, University of Washington, Seattle, Washington 98115, USA. spin ground states, ${ }^{3}$ theoretical and experimental results have established that an increase in the spin ground state is compensated by a corresponding decrease in total magnetic anisotropy. ${ }^{4}$ To overcome this problem, new approaches have emerged, such as using molecules containing a single paramagnetic lanthanide or actinide ion, both known for their often pronounced magnetic anisotropy. ${ }^{5,6}$ The effective energy barriers for magnetization reversal, $\Delta_{\text {eff }}$, for these molecules have been reported to exceed $1000 \mathrm{~K}\left(695 \mathrm{~cm}^{-1}\right){ }^{6}$ which is an order of magnitude larger than the original $\mathrm{Mn}_{12}$ SMM. ${ }^{2}$

More recently, it was shown that mononuclear first-row transition metal complexes can display significant magnetic anisotropy, originating from a near-orbitally degenerate ground state where the orbital angular momentum is left unquenched. ${ }^{7}$ Moreover, taking advantage of the Kramers theorem, ${ }^{8}$ fast quantum tunneling could be effectively avoided by using half-integer spin metal ions. Following this strategy, several mononuclear SMMs based on first-row transition metal ions were reported. ${ }^{9}$ For example, a recent linear two-coordinate $\mathrm{Fe}^{\mathrm{I}}$ complex displayed a $\Delta_{\text {eff }}$ of $354 \mathrm{~K}\left(246 \mathrm{~cm}^{-1}\right)$, and a magnetic hysteresis up to $6.5 \mathrm{~K}$, thereby challenging highperforming lanthanide systems. ${ }^{10}$ In addition to the synthetic challenges of generating such low-coordinate complexes, mononuclear SMMs of first-row transition metal ions have the intrinsic disadvantage of possessing weak spin-orbit coupling and thus may have a relatively small magnetic anisotropy. To meet this challenge, recent attention turned to $5 \mathrm{~d}$ metal ions, for which strong spinorbit coupling can be combined with the tunability of the electronic structure through the chemically controllable geometry and ligand field. ${ }^{11}$

Although SMM behavior seemed to be reserved for systems with $D<0$ (easy-axis type anisotropy; with $\hat{H}=D \hat{S}_{\mathrm{T}, z}{ }^{2}$ ), an increasing number of complexes featuring $D>0$ (easy-plane type anisotropy) in conjunction with SMM properties have been reported. ${ }^{12}$ However, for molecules with half-integer spin, a doubly degenerate ground state is always assured by the Kramers theorem, regardless of the sign of $D$. Thus, in principle, observation of slow magnetization dynamics via an Orbach process involving at least three $M_{S}$ microstates is always possible for a half-integer spin 
(a)

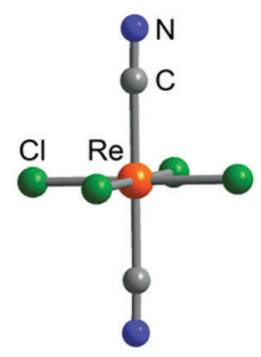

(b)

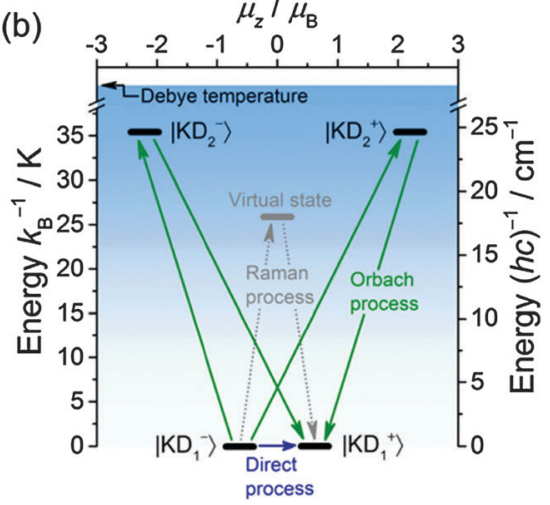

Fig. 1 (a) Structure of the trans- $\left[\mathrm{Re}^{\mathrm{IV}} \mathrm{Cl}_{4}(\mathrm{CN})_{2}\right]^{2-}$ complex obtained from single-crystal $\mathrm{X}$-ray diffraction on 1 at $50 \mathrm{~K}$. (b) Energy level diagram calculated from the previously determined $D$ and $E$ parameters. ${ }^{15 b}$ The blue shading is a schematic representation of the acoustic phonon density with the cut-off at the Debye temperature. The arrows indicate the direct (blue), Raman (grey), and Orbach (green) relaxation pathways from state $\left|\mathrm{KD}_{1}{ }^{-}\right\rangle$to $\left|\mathrm{KD}_{1}{ }^{+}\right\rangle$(the diagram is symmetrical for the reverse processes). The magnetic moment, $\mu_{z}$, is defined as $\mu_{z}=g_{\text {eff, } z} \mu_{\mathrm{B}} / 2$, where $g_{\text {eff }}$ is the $g$-factor treating the two doublets as effective spin $1 / 2$. The virtual state, relevant for the Raman process, is placed pictorially at an arbitrary energy with an arbitrary magnetic moment.

system, irrespective of the $M_{S}$ compositions of the ground-state doublet. ${ }^{13}$ Indeed, several SMMs with $D>0$ have been reported to exhibit this type of relaxation, despite a complete mismatch between the calculated and experimentally extracted energy barriers, ${ }^{12 c, e, f}$ thereby pointing towards the presence of alternative relaxation processes.

Recently, rhenium(Iv) complexes $\left(\mathrm{Bu}_{4} \mathrm{~N}\right)_{2}\left[\operatorname{Re}(\mathrm{ox}) \mathrm{X}_{4}\right]$ (ox = oxalate, $\mathrm{X}=\mathrm{Cl}, \mathrm{Br}),\left(\mathrm{PPh}_{4}\right)_{2}\left[\mathrm{ReF}_{6}\right] \cdot 2 \mathrm{H}_{2} \mathrm{O}$ and $\mathrm{Zn}(\mathrm{viz})_{4}\left[\mathrm{ReF}_{6}\right]$ (viz = 1-vinylimidazole) were shown to display slow relaxation of the magnetization with reported barriers of up to $29.6 \mathrm{~K}\left(20.6 \mathrm{~cm}^{-1}\right) .{ }^{14}$ Notably, for all four systems, the observed barrier is significantly lower than the expected value, as determined from the spin-Hamiltonian parameters. We previously reported on $\left(\mathrm{Bu}_{4} \mathrm{~N}\right)_{2}\left[\mathrm{Re}^{\mathrm{IV}} \mathrm{Cl}_{4}(\mathrm{CN})_{2}\right] \cdot 2 \mathrm{DMA}$ (1; Fig. 1a), ${ }^{15 a}$ and, subsequently, high-frequency and high-field electron paramagnetic resonance (HF-EPR) spectroscopic studies that allowed determination of its anisotropy parameters using the (zero-field) spin-Hamiltonian pertaining to $S=3 / 2$ given in eqn (1): $D / k_{\mathrm{B}}=16 \mathrm{~K}\left(+11 \mathrm{~cm}^{-1}\right)$ and $|E| / k_{\mathrm{B}}=4.6 \mathrm{~K}\left(3.2 \mathrm{~cm}^{-1}\right) .{ }^{15 b}$

$$
\hat{H}=D\left(\hat{S}_{z}^{2}-\frac{1}{3} S(S+1)\right)+E\left(\hat{S}_{x}^{2}-\hat{S}_{y}^{2}\right)
$$

Using these EPR-derived parameters, the ground $\left(\mathrm{KD}_{1}\right)$ and excited $\left(\mathrm{KD}_{2}\right)$ state Kramers doublet compositions are as follows:

$$
\begin{aligned}
& \left|\mathrm{KD}_{1}\right\rangle=-0.23| \pm 3 / 2\rangle+0.97|\mp 1 / 2\rangle \\
& \left|\mathrm{KD}_{2}\right\rangle= \pm 0.23|\mp 1 / 2\rangle \pm 0.97| \pm 3 / 2\rangle
\end{aligned}
$$

for which the admixture of the $M_{S}$ microstates arises from the transverse anisotropy (second term of eqn (1)) and the energy difference between $\mathrm{KD}_{1}$ and $\mathrm{KD}_{2}$ is $\Delta / k_{\mathrm{B}}=2\left(D^{2}+3 E^{2}\right)^{1 / 2} / k_{\mathrm{B}}=$ $35 \mathrm{~K}\left(25 \mathrm{~cm}^{-1}\right)$ (Fig. 1b).

Despite the positive value of $D$ and the predominant $M_{S}=$ $\pm 1 / 2$ character of the ground state Kramers doublet, $\mathrm{KD}_{1}$, we hereby report the observation of a slow relaxation of the magnetization for $\mathbf{1}$ that is reminiscent of SMM behavior, and discuss its possible origins. In 1, the rhenium(Iv) center of the $\left[\mathrm{ReCl}_{4}(\mathrm{CN})_{2}\right]^{2-}$ complex anion resides in an approximate $D_{4 \mathrm{~h}}$ symmetric environment ${ }^{15 a}$ and close inspection of the X-ray crystal structures at 50 and $139 \mathrm{~K}$ reveals only small differences in the local coordination environment of the metal ion. As expected for typical thermal contraction, the bond distances shrink slightly with decreasing temperature. The Re-Cl distances are 2.351(1) and 2.341(1) $\AA$ at $139 \mathrm{~K}$, while at $50 \mathrm{~K}$, these distances are 2.321(2) and 2.334(2) A, respectively. Selected interatomic distances and bond angles for $\mathbf{1}$ are listed in Table S1 (ESI $\dagger$ ).

Alternating current (ac) magnetic susceptibility measurements were performed to detect any slow relaxation of the magnetization in a polycrystalline sample of $\mathbf{1}$. In the absence of a static magnetic field, the relaxation time is too short to be observed in the experimentally accessible time window. Upon applying small static fields, $H$, however, clear frequency- and temperature-dependent ac signals were observed (Fig. 2 and Fig. S1, ESI $\dagger$ ). To elucidate the effect of the applied magnetic field on the slow relaxation of magnetization, the relaxation time was extracted from the variable-field and variable-temperature ac susceptibility data $(\tau(T, H)$; Fig. 3 and Fig. S1-S6, ESI $\dagger)$ by fitting the experimental data to the generalized Debye model. The relaxation time at $1.9 \mathrm{~K}$ monotonically decreases with increasing magnetic field (Fig. 3b). This behavior is expected for a direct process of relaxation, which involves only the two components of the lowestlying Kramers doublet and is accelerated upon increasing magnetic field (in accordance with the first term in eqn (2)). ${ }^{13,16}$ In the low field regime $\left(\mu_{\mathrm{B}} H \ll k_{\mathrm{B}} T\right)$, other processes (Orbach, Raman, etc.)

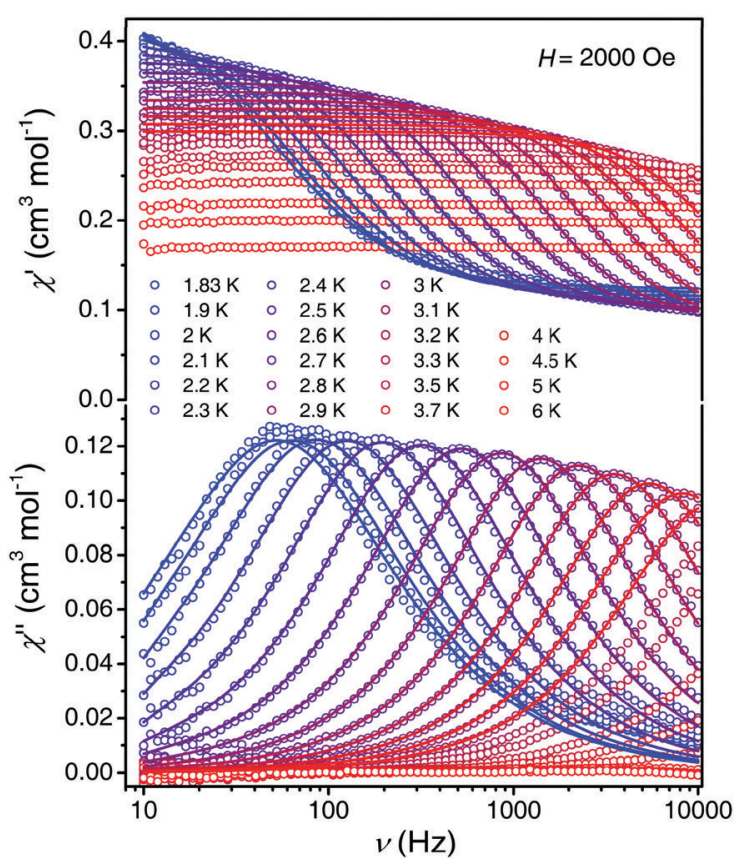

Fig. 2 Frequency dependence of the in-phase $\left(\chi^{\prime}\right.$, top) and out-of-phase ( $\chi^{\prime \prime}$, bottom) components of the ac magnetic susceptibility at different temperatures for a polycrystalline sample of 1 under a 2000 Oe dc field. The solid lines are the best fits to the generalized Debye model. 


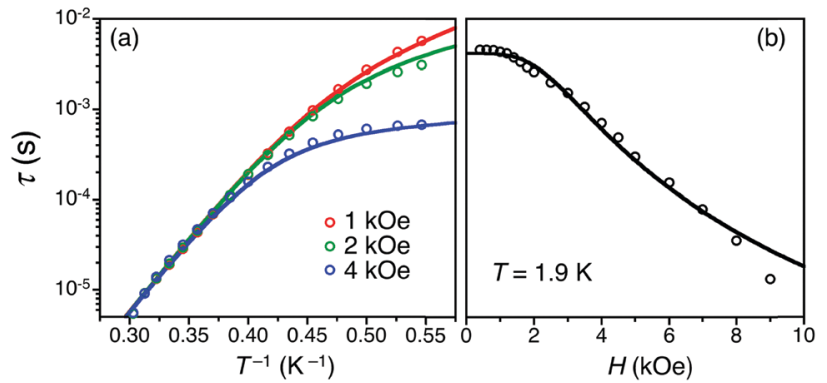

Fig. 3 Relaxation time $(\tau)$ versus (a) the inverse temperature $\left(T^{-1}\right)$ at $H=1$, 2 and $4 \mathrm{kOe}$ and versus (b) the applied dc field at $1.9 \mathrm{~K}$ up to $10 \mathrm{kOe}$ for 1 The solid lines are the best fits as described in the text.

governing magnetization relaxation are only weakly field dependent and thus their contributions to the relaxation time have been assumed to be constant, $k(T)$, as in eqn (2). ${ }^{12 b, 17,18}$

$$
\tau(H)^{-1}=A H^{4} T+k(T)
$$

Note that upon increasing the dc field, the probability of magnetization relaxation by quantum tunneling should be suppressed, thereby resulting in a slowing down of the relaxation. This effect is not experimentally observed for $\mathbf{1}$, and quantum tunnelling has thus not been considered in the modeling of its relaxation properties. ${ }^{16 d, 19,20}$

In order to reproduce the temperature dependence of the relaxation time, Orbach and/or Raman processes should be considered in addition to the direct process already evidenced in the field dependence (vide supra). ${ }^{17,21,22}$ Accordingly, two simple parameter-sparse models were considered, as given in eqn (3) and (4). These two approaches incorporate the contributions from both direct and Raman processes (eqn (3)) and both direct and Orbach processes (eqn (4)), with the fixed parameter $A=3.0 \times 10^{4} \mathrm{~s}^{-1} \mathrm{~K}^{-1} \mathrm{~T}^{-4}$ extracted from the $\tau(1.9 \mathrm{~K}, H)$ data.

$$
\begin{gathered}
\tau(T)^{-1}=A H^{4} T+C T^{n} \\
\tau(T)^{-1}=A H^{4} T+\tau_{0}{ }^{-1} \exp \left[-\Delta_{\mathrm{eff}} /\left(k_{\mathrm{B}} T\right)\right]
\end{gathered}
$$

Although the fit with eqn (3) is in good agreement with the experimental data (Fig. S7, ESI $\dagger$ ), the Raman exponent, $n$, reaches a suspiciously large value of $12 \pm 1$, which significantly exceeds the expected value of $n=9$ for a Kramers system. ${ }^{21}$ Nevertheless, it should be noted that examples of $n>9$ have been reported previously. ${ }^{23}$ These results were later attributed to the presence of a particular energy level structure where the energy of the first excited state, $\Delta$, was close to, but larger than, the Debye temperature of the lattice (see Fig. 1b; in the present case, the Debye approximation is here justified by the low energy of the first excited state $\left.{ }^{24}\right)$. In this case, the Raman expression for $\tau(T)^{-1}$ is given by eqn (5). ${ }^{21 a}$

$$
\tau(T)^{-1}=C\left(T^{9}+180\left(\frac{k_{\mathrm{B}}}{\Delta}\right)^{2} T^{11}+3240\left(\frac{k_{\mathrm{B}}}{\Delta}\right)^{4} T^{13}+\ldots\right)
$$

For 1 with $\Delta / k_{\mathrm{B}}=35 \mathrm{~K}\left(25 \mathrm{~cm}^{-1}\right)$, the prefactors of the $T^{11}$ and $T^{13}$ terms amount to only $0.15 \mathrm{~K}^{-2}$ and $2.2 \times 10^{-3} \mathrm{~K}^{-4}$, respectively, indeed indicating an expected dominant $T^{9}$ behavior in the measured temperature window. On the other hand, the model containing an Orbach process (eqn (4)), which necessitates $\Delta$ to be equal to or lower than the Debye temperature, ${ }^{13}$ is also insufficient to reproduce the experimental relaxation time (Fig. S8, ESI $\dagger$ ). Furthermore, the calculated parameter $k\left(154 \mathrm{~s}^{-1}\right.$ at $\left.1.9 \mathrm{~K}\right)$ obtained from the fitting parameters of eqn (4) is in disagreement with the one obtained from eqn (2) and the field-dependence of the relaxation time $\left(k=226 \mathrm{~s}^{-1}\right.$ at $\left.1.9 \mathrm{~K}\right)$. The disagreement between these models strongly suggests the presence of more than two types of relaxation processes in $\mathbf{1}$. Consequently, the direct, Raman and Orbach processes were all included in the fitting model given by eqn (6).

$$
\tau(T, H)^{-1}=A H^{4} T+C T^{n}+\tau_{0}{ }^{-1} \exp \left[-\Delta_{\mathrm{eff}} /\left(k_{\mathrm{B}} T\right)\right]
$$

In order to improve the reliability of the model, the complete experimental set of relaxation time data as a function of both field and temperature, $\tau(T, H)$, was fitted simultaneously using eqn (6). The resulting fit provided good agreement with all data, as depicted graphically with the solid lines in Fig. 3, and afforded $A=2.9 \times 10^{4} \mathrm{~s}^{-1} \mathrm{~K}^{-1} \mathrm{~T}^{-4}, C=2.5 \mathrm{~s}^{-1} \mathrm{~K}^{-6.9}, n=6.9$, $\tau_{0}=5.7 \times 10^{-11} \mathrm{~s}$, and $\Delta_{\mathrm{eff}} / k_{\mathrm{B}}=39 \mathrm{~K}\left(27 \mathrm{~cm}^{-1}\right)$. This result supports the relevance of direct, Raman, and Orbach processes for the magnetization dynamics of $\mathbf{1}$ in the temperature (Fig. S9, $\mathrm{ESI} \dagger$ ) and magnetic field ranges investigated. Considering the two Kramers doublets, $\mathrm{KD}_{1}$ and $\mathrm{KD}_{2}$ discussed above, the transverse anisotropy (eqn (1)) and the nonvanishing integral of $\left\langle \pm 3 / 2\left|E\left(\hat{S}_{x}^{2}-\hat{S}_{y}^{2}\right)\right| \mp 1 / 2\right\rangle$ enable both Orbach and Raman mechanisms of relaxation. ${ }^{13}$ Indeed, the energy separation between $\mathrm{KD}_{1}$ and $\mathrm{KD}_{2}\left(\Delta=2\left(D^{2}+3 E^{2}\right)^{1 / 2}\right)$, estimated to $35 \mathrm{~K}$ $\left(25 \mathrm{~cm}^{-1}\right)$ from HF-EPR data, is in good agreement with the barrier of $39 \mathrm{~K}\left(27 \mathrm{~cm}^{-1}\right)$ determined for the Orbach process from the ac susceptibility data.

Spectroscopy offers an opportunity to access the energy levels involved in the Orbach relaxation, but has rarely been employed in directly determining the energy level structure in mononuclear SMMs. ${ }^{9 b, 12 d, 14 b}$ In an attempt to confirm the energy level structure of $\mathbf{1}$ (Fig. 1b) in the absence of a perturbing magnetic field, inelastic neutron scattering (INS) spectroscopy was performed on a polycrystalline sample. We were, however, unable to observe the presence of any excited state in the energy window from $\mathrm{ca}$. 10 to $50 \mathrm{~cm}^{-1}$ (14 to $72 \mathrm{~K}$; Fig. S10 and S11, ESI $\dagger$ ), possibly due to the high content of ${ }^{1} \mathrm{H}$ that gives rise to an intense spectral background. Interestingly, analysis of the energy dependence of the phonon spectrum affords a Debye temperature of $\theta_{\mathrm{D}} \approx 139 \mathrm{~K}$ (Fig. S12, ESI $\dagger$ ), suggesting the applicability of the Debye model in the studied temperature regime. To circumvent the problem of the strong spectral background, frequency-domain Fourier-transform THz-EPR (FD-FT THz-EPR) spectroscopy was employed in detecting EPR transitions in the energy range of $c a .10$ to $30 \mathrm{~cm}^{-1}$ (14 to $43 \mathrm{~K}$; Fig. S13 and S14, ESI $\dagger$ ). Unfortunately, no magnetic excitations could be detected here either, preventing the confirmation of the energy level diagram derived from the preceding high-field EPR spectroscopic study.

The foregoing results demonstrate the necessity of invoking Orbach, direct, and Raman-type processes in explaining the 
multifaceted magnetization dynamics observed in the mononuclear complex salt $\left(\mathrm{Bu}_{4} \mathrm{~N}\right)_{2}\left[\mathrm{Re}^{\mathrm{IV}} \mathrm{Cl}_{4}(\mathrm{CN})_{2}\right] \cdot 2 \mathrm{DMA}$. Whilst this multi-parameter description of $\tau(T, H)$ was only satisfactory after introduction of the Orbach mechanism, the lack of observation of the relevant excited state by spectroscopic techniques brings justified doubts on the presence of an operative Orbach relaxation in 1. This original example highlights the complexity of describing paramagnetic relaxation in molecular systems. Even if these elaborate multi-mechanism models for describing the relaxation phenomena are now becoming fashionable in the field of molecular magnetism, the combination of a large number of parameters with relatively uncharacteristic $\tau(T, H)$ data in a narrow temperature interval may lead to questionable conclusions.

This work was supported by the National Science Foundation (NSF) under Grant CHE-1464841, the CNRS (PICS No. 06485), the University of Bordeaux, the Conseil Regional d'Aquitaine, the ANR, the French Embassy in the US (Chateaubriand fellowship for X. F.), the GdR MCM-2 and Sun Yat-Sen University (the International Program of Project 985 for J.-L. L.). K. S. P. thanks the Danish Research Council for Independent Research for a DFF-Sapere Aude Research Talent grant (409000201). The low temperature crystal structure was collected at the Stanford Nano Shared Facilities (SNSF), supported by the NSF under award ECCS-1542152. We thank also Philip C. Bunting for helpful discussions.

\section{Notes and references}

1 G. Gatteschi, R. Sessoli and J. Villain, Molecular Nanomagnets, Oxford University Press, Oxford, 2006.

2 (a) R. Sessoli, H. L. Tsai, A. R. Schake, S. Wang, J. B. Vincent, K. Folting, D. Gatteschi, G. Christou and D. N. Hendrickson, J. Am. Chem. Soc., 1993, 115, 1804; (b) R. Sessoli, D. Gatteschi, A. Caneschi and M. A. Novak, Nature, 1993, 365, 141.

3 C. J. Milios, A. Vinslava, W. Wernsdorfer, S. Moggach, S. Parsons, S. P. Perlepes, G. Christou and E. K. Brechin, J. Am. Chem. Soc., 2007, 129, 2754.

4 (a) F. Neese and E. I. Solomon, Inorg. Chem., 1998, 37, 6568; (b) O. Waldmann, Inorg. Chem., 2007, 46, 10035; (c) E. Ruiz, J. Cirera, J. Cano, S. Alvarez, C. Loose and J. Kortus, Chem. Commun., 2008, 52; (d) F. Neese and D. A. Pantazis, Faraday Discuss., 2011, 148, 229.

5 (a) N. Ishikawa, M. Sugita, T. Ishikawa, S. Y. Koshihara and Y. Kaizu, J. Am. Chem. Soc., 2003, 125, 8694; (b) F. Branzoli, P. Caretta, M. Filibian, G. Zoppellaro, M. J. Graf, J. R. Galan-Mascaros, O. Fuhr, S. Brink and M. Ruben, J. Am. Chem. Soc., 2009, 131, 4387; (c) S.-D. Jiang, B.-W. Wang, H.-L. Sun, Z.-M. Wang and S. Gao, J. Am. Chem. Soc., 2011, 133, 4730; (d) J.-L. Liu, Y.-C. Chen, Y.-Z. Zheng, W.-Q. Lin, L. Ungur, W. Wernsdorfer, L. F. Chibotaru and M.-L. Tong, Chem. Sci., 2013, 4, 3310; (e) J. D. Rinehart and J. R. Long, J. Am. Chem. Soc., 2009, 131, 12558; $(f)$ K. R. Meihaus, J. D. Rinehart and J. R. Long, Inorg. Chem., 2011, 50, 8484; $(g)$ M. Gregson, N. F. Chilton, A.-M. Ariciu, F. Tuna, I. F. Crowe, W. Lewis, A. J. Blake, D. Collison, E. J. L. McInnes, R. E. P. Winpenny and S. T. Liddle, Chem. Sci., 2016, 7, 155; (h) J. J. Baldoví, S. Cardona-Serra, J. M. Clemente-Juan, E. Coronado and A. Gaita-Arino, Chem. Sci., 2013, 4, 938; (i) L. Ungur, J. J. Le Roy, I. Korobkov, M. Murugesu and L. F. Chibotaru, Angew. Chem., Int. Ed., 2014, 53, 4413.
6 J. Liu, Y.-C. Chen, J.-L. Liu, V. Vieru, L. Ungur, J.-H. Jia, L. F. Chibotaru, Y. Lan, W. Wernsdorfer, S. Gao, X.-M. Chen and M.-L. Tong, J. Am. Chem. Soc., 2016, 138, 5441.

7 D. E. Freedman, W. H. Harman, T. D. Harris, G. J. Long, C. J. Chang and J. R. Long, J. Am. Chem. Soc., 2010, 132, 1224.

8 H. A. Kramers, Proc. R. Acad. Sci. Amsterdam, 1930, 33, 959.

9 (a) G. A. Craig and M. Murrie, Chem. Soc. Rev., 2015, 44, 2135; (b) Y. Rechkemmer, F. D. Breitgoff, M. van der Meer, M. Atanasov, M. Hakl, M. Orlita, P. Neugebauer, F. Neese, B. Sarkar and J. van Slageren, Nat. Commun., 2016, 7, 10467.

10 (a) J. M. Zadrozny, D. J. Xiao, M. Atanasov, G. J. Long, F. Grandjean, F. Neese and J. R. Long, Nat. Chem., 2013, 5, 577; (b) J. M. Zadrozny, D. M. Xiao, J. R. Long, M. Atanasov, F. Neese, F. Grandjean and G. J. Long, Inorg. Chem., 2013, 52, 13123.

11 (a) X.-Y. Wang, C. Avendaño and K. R. Dunbar, Chem. Soc. Rev., 2011, 40, 3213; (b) K. S. Pedersen, J. Bendix and R. Clérac, Chem. Commun., 2014, 50, 4396; (c) S. K. Singh and G. Rajaraman, Nat. Commun., 2016, 7, 10669.

12 (a) J. M. Zadrozny, J. Liu, N. A. Piro, C. J. Chang, S. Hill and J. R. Long, Chem. Commun., 2012, 48, 3927; (b) S. Gómez-Coca, A. Urtizberea, E. Cremades, P. J. Alonso, A. Camón, E. Ruiz and F. Luis, Nat. Commun., 2014, 5, 4300; (c) S. Gómez-Coca, E. Cremades, N. Aliaga-Alcalde and E. Ruiz, J. Am. Chem. Soc., 2013, 135, 7010; (d) E. Colacio, J. Ruiz, E. Ruiz, E. Cremades, J. Krzystek, S. Carretta, J. Cano, T. Guidi, W. Wernsdorfer and E. K. Brechin, Angew. Chem., Int. Ed., 2013, 52, 9130; (e) S. Vaidya, A. Upadhyay, S. K. Singh, T. Gupta, S. Tewary, S. K. Langley, J. P. S. Walsh, K. S. Murray, G. Rajaraman and M. Shanmugam, Chem. Commun., 2015, 51, 3739; $(f)$ J. Vallejo, I. Castro, R. RuizGarcia, J. Cano, M. Julvet, F. Lloret, G. De Munno, W. Wernsdorfer and E. Pardo, J. Am. Chem. Soc., 2012, 134, 15704.

13 R. Orbach, Proc. R. Soc. London, Ser. A, 1961, 264, 458.

14 (a) J. Martinez-Lillo, T. F. Mastropietro, E. Lhotel, C. Paulsen, J. Cano, G. Di Munno, J. Faus, F. Lloret, M. Julve, S. Nellutia and J. Krzystek, J. Am. Chem. Soc., 2013, 135, 13737; (b) K. S. Pedersen, M. Sigrist, M. A. Sørensen, A.-L. Barra, T. Weyhermüller, S. Piligkos, C. Aa. Thuesen, M. G. Vinum, H. Mutka, H. Weihe, R. Clérac and J. Bendix, Angew. Chem., Int. Ed., 2014, 53, 1351.

15 (a) T. D. Harris, M. V. Bennet, R. Clérac and J. R. Long, J. Am. Chem. Soc., 2010, 132, 3980; (b) X. Feng, J. Liu, T. D. Harris, S. Hill and J. R. Long, J. Am. Chem. Soc., 2012, 134, 7521.

16 (a) R. Orbach, Proc. R. Soc. London, Ser. A, 1961, 264, 485; (b) T. L. Bohan and H. J. Stapleton, Phys. Rev., 1969, 182, 385; (c) K. N. Shrivastava, Phys. Status Solidi B, 1983, 117, 437; (d) J. M. Zadrozny, M. Atanasov, A. M. Bryan, C.-Y. Lin, B. D. Rekken, P. P. Power, F. Neese and J. R. Long, Chem. Sci., 2013, 4, 125.

17 A. Abragam and B. Bleaney, Electron Paramagnetic Resonance of Transition Ions, Dover, New York, 1986.

18 See for example: (a) I. Chiorescu, W. Wernsdorfer, A. Müller, H. Bögge and B. Barbara, Phys. Rev. Lett., 2000, 84, 3454; (b) K. S. Pedersen, J. Dreiser, H. Weihe, R. Sibille, H. V. Johannesen, M. A. Sørensen, B. E. Nielsen, M. Sigrist, H. Mutka, S. Rols, J. Bendix and S. Piligkos, Inorg. Chem., 2015, 54, 7600; (c) L. Tesi, E. Lucaccini, I. Cimatti, M. Perfetti, M. Mannini, M. Atzori, E. Morra, M. Chiesa, A. Caneschi, L. Sorace and R. Sessoli, Chem. Sci., 2016, 7, 2074; (d) M. Atzori, L. Tesi, E. Morra, M. Chiesa, L. Sorace and R. Sessoli, J. Am. Chem. Soc., 2016, 138, 2154.

19 E. Lucaccini, L. Sorace, M. Perfetti, J.-P. Costes and R. Sessoli, Chem. Commun., 2014, 50, 1648.

20 A. Fort, A. Rettori, J. Villain, D. Gatteschi and R. Sessoli, Phys. Rev. Lett., 1998, 80, 612.

21 (a) K. N. Shrivastava, Phys. Status Solidi B, 1972, 51, 377; (b) A. Singh and K. N. Shrivastava, Phys. Status Solidi B, 1979, 95, 273.

22 C. B. P. Finn, R. Orbach and W. P. Wolf, Proc. Phys. Soc., London, 1961, 77, 261.

23 A. Kiel and W. B. Mims, Phys. Rev., 1967, 161, 386.

24 (a) S. S. Eaton and G. R. Eaton, Biol. Magn. Reson., 2000, 19, 29; (b) S. T. Liddle and J. van Slageren, Chem. Soc. Rev., 2015, 44, 6655. 